\title{
De quelques signes de main dans le cinéma d'animation français des premiers temps
}

The filming of the filmmaker's hands in early French animation

\section{Valérie Vignaux}

\section{(2) OpenEdition \\ Journals}

Édition électronique

URL : https://journals.openedition.org/1895/3920

DOI : 10.4000/1895.3920

ISBN : 978-2-8218-0984-0

ISSN : $1960-6176$

Éditeur

Association française de recherche sur l'histoire du cinéma (AFRHC)

Édition imprimée

Date de publication : 1 décembre 2009

Pagination : $96-107$

ISBN : 978-2-913758-60-5

ISSN : 0769-0959

\section{Référence électronique}

Valérie Vignaux, «De quelques signes de main dans le cinéma d'animation français des premiers temps », 1895. Mille huit cent quatre-vingt-quinze [En ligne], 59 | 2009, mis en ligne le 01 décembre 2012, consulté le 17 avril 2022. URL : http://journals.openedition.org/1895/3920 ; DOI : https://doi.org/ $10.4000 / 1895.3920$ 

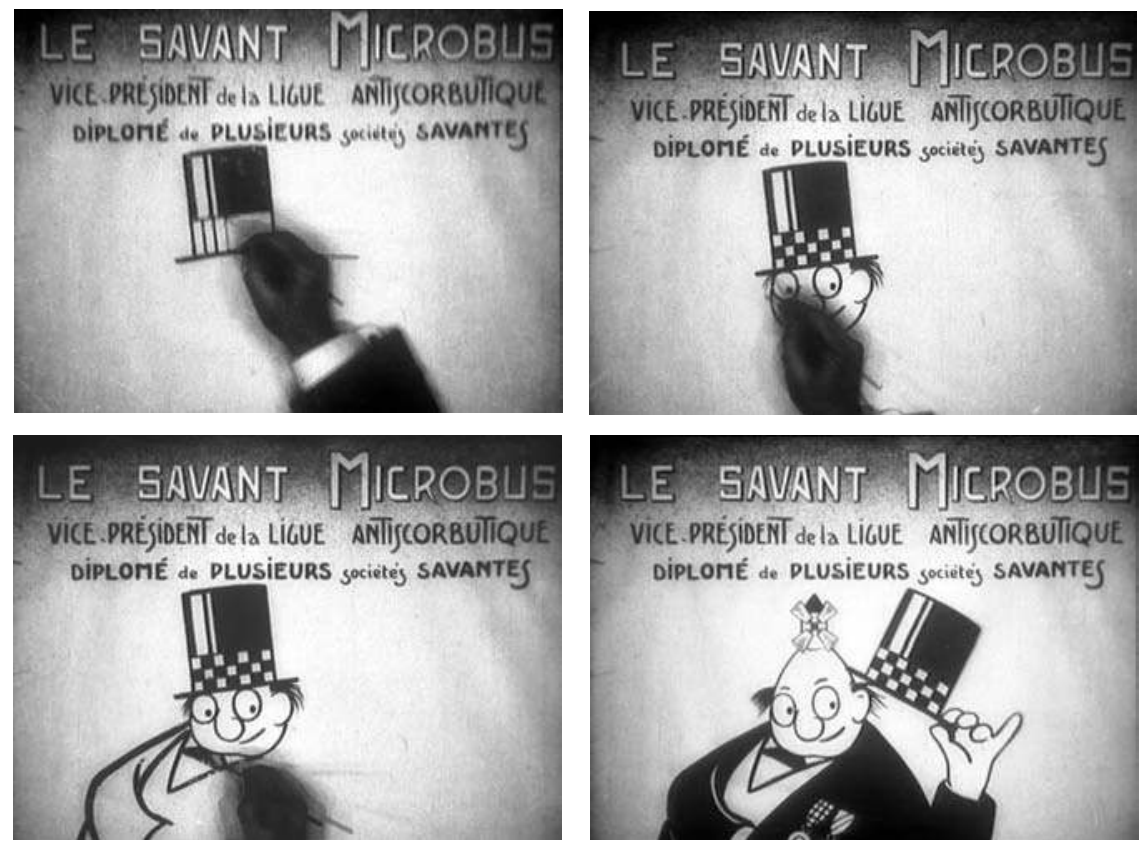

Robert Lortac, la Maison automatique (1921). 


\section{De quelques signes de main dans le cinéma d'animation français des premiers temps}

par Valérie Vignaux

Les cinéastes d'animation des premiers temps introduisent volontiers leurs mains dans le champ de l'objectif. Ce curieux dispositif se découvre dans les films d'Émile Cohl (Fantasmagorie - 1908, les Fantaisies d'Agénor Maltracé - 1911), mais aussi chez Marius O'Galop (On doit le dire -1919, Bécassote à la mer - 1920, le Lièvre et la tortue - 1921) ou Robert Lortac ( la Maison automatique - 1921, le Canard en ciné - 1922). Procédé qui n'est pas spécifiquement français puisqu'on le retrouve de l'autre côté de l'Atlantique chez Winsor MacCay (Gertie le dinosaure - 1914) ou dans les premiers films de Walt Disney (Alice in cartoonland 1923). Récurrent de 1908 à $1923^{1}$ probablement, le motif de la main pourrait être envisagé comme le marqueur d'une compréhension partagée des enjeux du cinéma d'animation naissant, et l'étude de ses manifestations permettrait de les restituer. On ne s'intéressera toutefois, dans le cadre de cet article, qu'aux mains d'Émile Cohl, de Marius O'Galop ou de Robert Lortac.

Du «truc» à l'art

Le cinéma d'animation a tout d'abord été présenté comme un «truc ${ }^{2}$, une mise en mouvement «magique» de l'inanimé et les films d'Émile Cohl côtoyaient dans les catalogues ceux de Georges Méliès. Reçu par le Ciné-Club de France pour une conférence en juin 1920, Émile Cohl déclarait en préambule: «Les dessins animés font partie des bandes de cinéma constituées au moyen de trucs». En 1925, dans l'article qu'il fait paraître sous pseudonyme dans le

1 Osvaldo Cavandoli reprendra le procédé dans sa série intitulée la Linea (1972).

2 Certaines des réflexions présentes dans ce texte ont fait l'objet d'une première élaboration dans le cadre d'une journée d'études organisée par Laurent Mannoni et Bernard Bénoliel: «Trucs, trucages et effets spéciaux, de Méliès à Spielberg ", Cinémathèque française, 2 juin 2008. 
Larousse mensuel, il le souligne encore: "À côté du cinématographe ordinaire montrant la vie dans ses multiples manifestations, on put voir, presque aussitôt, le cinématographe truqué, ou mieux à trucs. Les dessins animés forment une variété de cette catégorie » ${ }^{3}$. Pourtant lorsqu'il écrit ce texte en 1925, les «trucs » cinématographiques sont tombés en désuétudes car la divulgation dans la presse du procédé d'image par image - ou d'arrêt caméra - a conduit à sa banalisation4. En 1922, Étienne Arnaud, qui fut proche d'Émile Cohl, puisqu'ils co-réalisèrent ensemble plusieurs films, en témoignait:

Avant qu'il existât même des revues ou journaux cinématographiques, tous les articles parus sur le cinéma dans les grands périodiques ou dans les feuilles populaires avaient pour sujet: les trucs

$1895 /$ du cinéma. Des reporters à court de copie, ont fait, aux dépens de l'illusion et des auteurs de scénarios des «papiers » à épisodes sans fin, sur la cuisine de tous les sujets que la projection rend sensationnels. Et les livres de vulgarisation ont disséqué les ombres vivantes, trépané les auteurs qui se croyaient encore un peu d'or dans leurs cervelles et montré au microscope leurs... cellulos grossis mille fois 5 .

La présentation de la main, parce qu'elle rappelle la présence du dessinateur, de l'artiste, permet de distinguer le film de la technique et ainsi l'affranchit du «truc». Le spectateur «pris par la main » - est convié à partager un univers singulier ou autrement dit une œuvre. En ce début des années 1920, les paradigmes sollicités pour conforter le cinéma ont manifestement relégué les aspects techniques, alors qu'un temps, sous le mode de l'«invention », ils semblaient primordiaux. Le désarroi d'Henri Focillon, lorsqu'il en vient à évoquer la photographie, en conclusion de son Éloge de la main, montre les ambiguïtés que le photosensible - une technique d'enregistrement - entretient avec l'art (Henri Focillon dont on sait par ailleurs les engagements institutionnels ${ }^{6}$ en faveur du cinéma - mais pédagogique):

Une perception pure, dépouillée de substance et de densité, ou plutôt un éblouissant souvenir optique, fixé dans cette mémoire cristalline qui retient tout, la chambre noire. La matière, la

3 Émile Cohl, «Les dessins animés et à trucs », le Journal du ciné-club, 18 juin 1920 et J.-B. de Tronquières [Émile Cohl] «Dessins animés », Larousse mensuel n²22, août 1925. Ces deux textes sont reproduits dans Valérie Vignaux (dir.), "Émile Cohl», 1895, n53, décembre 2007.

4 Cf. Roland Cosandey, "Cinéma 1908, films à trucs et Film d'Art: une campagne de l'Illustration », Cinémathèque $n^{\circ} 3$, printemps-été 1993, pp. 58-71.

5 Étienne Arnaud et Boisyvon, Le Cinéma pour tous, Paris, Garnier, 1922, p. 74.

6 Cf. Valérie Vignaux, "Henri Focillon et le cinéma, une archéologie du documentaire sur l'art », dans

P.-H. Frangne, G. Mouëllic et C. Viart (dir. ), Filmer I'acte de création, Rennes, PUR, 2009, pp. 161-168. 
main, l'homme même sont absents. Ce vide absolu dans la totalité de la présence est chose étrange. Peut-être ai-je sous les yeux l'exemple d'une poétique future: je ne puis peupler encore ce silence et ce désert? ${ }^{7}$.

Aux lendemains de la Première Guerre, les personnalités agissantes s'efforcent de légitimer le spectacle cinématographique, en convoquant le théâtre, la littérature ou les arts et déclarent volontiers que s'il ne peut être artistique, qu'il soit au moins pédagogique; on demande d'ailleurs au cinéma instructif de revêtir les atours de l'art... Émile Cohl, comme d'autres, y voit même le seul avenir possible et conclut son article de 1925 par ces mots aux accents prophétiques:

À part des fantaisies amusantes, réservées, semble-t-il, en ce moment, à la publicité, il est bien certain que c'est dans le côté instructif, que les dessins animés trouveront leur avenir, et ils deviendront de plus en plus les adjuvants clairs et précis de la parole des maîtres8.

Les cinéastes d'animation français n'ont donc pas été écartés des débats, bien au contraire. Ils ont même été invités à collaborer à l'exposition de 1924, qui se tient au Musée Galliera, intitulée "l'Art dans le cinéma français», et qui fut un moment important quant à la reconnaissance du medium: «première exposition [consacrée à] une invention qui, en si peu de temps, s'est élevée jusqu'à la dignité, jusqu'à la grandeur de l'Art» 9 . Manifestation composée de deux sections: "rétrospective » et "enseignement » et qui vise surtout, il me semble, à distinguer les personnalités éligibles au rang d'artistes tels Abel Gance, Marcel I'Herbier ou Germaine Dulac. Or, aux côtés des esthètes on trouve les animateurs, soit dans l'ordre du catalogue: I' «Atelier Lortac, Cohl, Cheval, Jodelet, Landelle, Leyritz, Lortac, Mourlan, O'Galop, Pinchon et Publi-Ciné (voir Lortac) »; cinéastes qui exposent en très grande majorité, des dessins exécutés dans le cadre de la commande publicitaire, et distribués par Publi-Ciné10.

La pratique du cinéma d'animation n'est donc pas sans contradictions: parce qu'ils emploient le cinéma image par image, leur art est le produit d'une technique, mais parce qu'ils sont dessinateurs on peut les apparenter aux artistes; or, ils exercent dans le cadre de la requête

7 Henri Focillon, «Éloge de la main », dans Vie des formes, Paris, Presses universitaires de France, 1984, p. 126. Je souligne.

$8 \mathrm{~J}$.-B. de Tronquières [Émile Cohl] «Dessins animés», art. cit.

9 Georges Lecomte, "Un art nouveau », Musée Galliera, Exposition de l'Art dans le cinéma français, catalogue, 1924, p. 1.

10 lbid, pp. 10-12. 
1895 /

$n^{\circ} 59$

décembre

2009
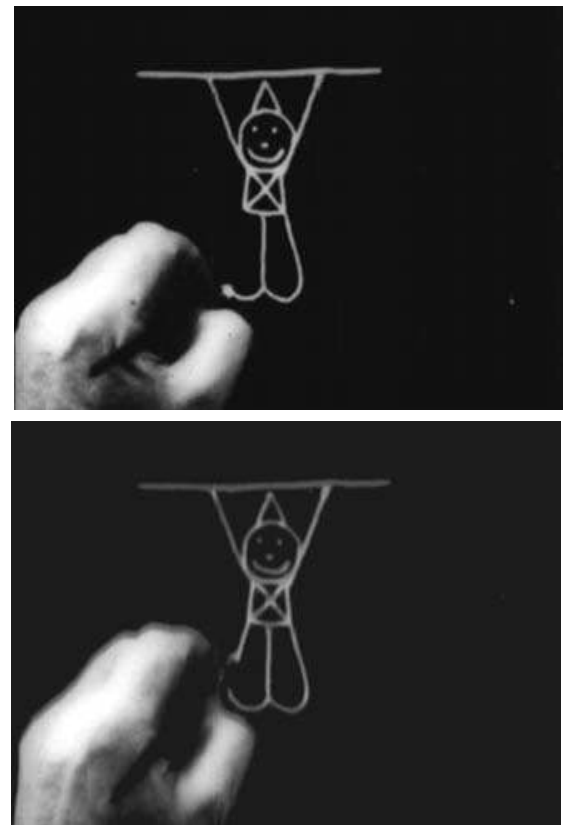

industrielle et les voilà soumis au commerce. Autant de paradoxes qui expliquent le recours au cinéma instructif car il constitue à l'évidence un modèle de reconnaissance assez aisé.

\section{Jeux de mains}

La présence de la main a une fonction métonymique. Pour Henri Focillon, elle est à la fois l'instrument et l'œuvre: "l'outil [la main] en soi n'est pas moins remarquable que l'usage auquel on le destine, il est à lui seul valeur et résultat " ${ }^{11}$. La main prolonge l'esprit et suivre son cheminement conduit à percevoir l'art de manière intime car en retrouvant les gestes de l'artiste, on partage les mystères de la création: "les mains sont l'instrument de la création, mais d'abord l'organe de la connaissance [...]. Elles sont presque des êtres animés. [...] La main est action: elle prend, elle crée, et parfois on dirait qu'elle pense ${ }^{12}$.

Si les mains d'Émile Cohl, de Marius O'Galop ou de Robert Lortac, apparaissent à l'écran extrêmement soignées - les animateurs ont en effet revêtu pour l'occasion leurs plus beaux vêtements, arborant chemises blanches et boutons de manchette - pour autant, les univers qu'elles convoquent sont dissemblables. La main d'Émile Cohl est celle du magicien qui exécute sous nos yeux des tours: le bout de bois s'anime et devient personnage dans Fantasmagorie, ou encore de l'œuf surgit un monde dans les Beaux-arts mystérieux. Compréhension du geste qui est manifeste et explicite, Étienne Arnaud en témoigne: "le metteur en scène de films de ce genre ou pour mieux dire I' "animateur" de ces

11 Henri Focillon, «Éloge de la main», dans Vie des formes, op. cit., p. 110.

12 Ibid, p. 103. 
objets inertes doit se munir d'une belle dose de patience et être doué d'une habileté de doigts qui rappelle celle des prestidigitateurs " ${ }^{13}$. Émile Cohl, illusionniste à l'instar de Georges Méliès, s'attache pourtant à différencier sa pratique de celle du magicien de Montreuil. Dans le Binettoscope, film où il semble lui rendre hommage, nous voyons un personnage revêtu d'un costume de clown accomplir les gestes rituels du tour de magie, or il lance sur un écran dressé au second plan, des notes de musique dessinées qui soudain se métamorphosent en visages (en "binettes»). La gestuelle est comparable à celle employée par Georges Méliès dans le Mélomane où il projetait sur une portée dessinée en haut du cadre, autant de têtes à son image, converties ainsi en notes de musique. Pour Donald Crafton, le renvoi à la prestidigitation serait spécifique à Cohl14, et selon lui, elle le différencierait des animateurs américains qui se réfèreraient au théâtre ou à l'expérience de la scène:

Nombreux sont les points communs entre l'iconographie des premiers films à trucs et l'œuvre de Cohl. Le motif familier de la main de l'artiste en gros plan est aussi un des favoris de Cohl et le spectateur, à de nombreuses reprises, voit par exemple
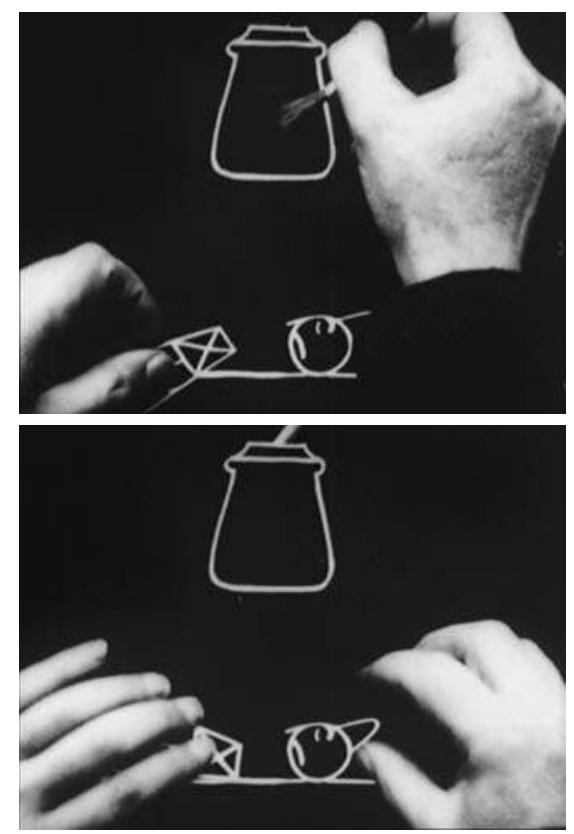

son doigt entrer dans le champ de l'objectif, comme lorsqu'il bouscule l'ivrogne dans le Rêve d'un garçon de café. Mais l'intention de Cohl diffère des premiers films d'animation où cela avait le sens d'une performance scénique et où les dessins étaient secondaires à l'artiste comme acteur. Cohl introduit sa présence dans ses films, non pas tant par son autonomie corporelle, que par la rigueur de son style et son univers esthétique unique. C'est comme si l'attention du spectateur était détournée par le magicien, à travers les mécanismes du truc ${ }^{15}$.

13 Étienne Arnaud et Boisyvon, Le Cinéma pour tous, Paris, Garnier, 1922, p. 71.

14 Cohl, adolescent, a été l'assistant d'un magicien, cf. Valérie Vignaux (dir.), "Émile Cohl », op. cit., p. 21. 15 Donald Crafton, Before Mickey, the animated film 1898-1928, Cambridge/Londres, The Mit press, 1984, pp. 87-88. 
La relation entre théâtre et animation n'est pourtant pas inconnue de Cohl qui se souvenait avoir assisté à une présentation de Gertie le dinosaure par Winsor McCay, lors de son séjour aux États-Unis. La description qu'il en fait, montre qu'il perçoit très justement les enjeux du spectacle: le dandysme de l'illustrateur qui vise à valoriser le numéro afin de le distinguer des représentations populaires, tout en l'affiliant à une tradition où l'élégance est le style des maîtres d'œuvre, soit le magicien, le chef d'orchestre ou monsieur Loyal. Il souligne encore, I'interactivité entre le créateur et la créature, tout en rappelant la supériorité ou la directivité, du premier sur la seconde.

Les films de Winsor McCay étaient dessinés d'une façon admirable; mais une des principales causes de leur succès consistait dans la manière de les présenter au public. Je me souviens d'avoir assisté à une de ces représentations publiques au théâtre Hammerstein, à New-York. Le principal, je pourrais dire l'unique personnage de la bande était un animal antédiluvien, une sorte de diplodocus monstrueusement grand. Au commencement le tableau représentait un arbre et des rochers. Sur la scène, devant l'écran, Winsor McCay, très élégant, armé d'un court fouet de chien prononçait un speech puis tel un dompteur ou M. Loyal, au Cirque, il appelait la bête qui surgissait des rochers. Alors, et toujours au commandement de l'artiste c'était tout un travail de haute école qui se déroulait; I'animal dansant, tournant et finissant par avaler arbres, rochers et venant à la fin s'agenouiller devant le public qui pouvait à la fois applaudir l'œuvre et l'artiste ${ }^{16}$.

L'œuvre de Cohl est plus éclectique que celle de Winsor McCay, mais les références au scénique ou au théâtral n'en sont pas absentes; on peut citer ses adaptations comme le Tout petit Faust ou le Petit Chantecler et rappeler qu'il fut acteur ${ }^{17}$ au Grand-Guignol. Par contre, il est vrai qu'il excelle, lorsqu'il puise les ressources de l'inventivité dans un univers propre, intime, et fait de réemploi ${ }^{18}$. Lorsqu'il fait surgir du merveilleux à partir du dérisoire, car «en prenant dans sa main quelques déchets du monde, I'homme a pu en inventer un autre qui est tout de lui »19.

Les mains de Marius O'Galop ou de Robert Lortac ne sont plus celles du magicien, pourtant lorsqu'elles interviennent dans les films, elles soulignent leurs parentés avec celles d'Émile Cohl. Marius O'Galop dans On doit le dire fait surgir sous nos yeux les traits d'un personnage

16 Description reproduite dans Émile Cohl, "Les dessins animés et à trucs», art. cit. et dans Étienne Arnaud et Boisyvon, op. cit., p. 82. Je souligne.

17 Cf. Olivier Goetz, Isabelle Moindrot, Romain Piana, «Émile Cohl et le théâtre», dans Valérie Vignaux (dir.), «Émile Cohl», op. cit., pp. 87-96.

18 On reconnaît par exemple une de ses têtes de pipe (titre d'un livre de L.-G. Mostraille qu'il a illustré de photographies, Paris, Vannier, 1885) dans les Générations comiques; le serpent de Mon ami Polichinelle (un album pour enfant illustré en 1888), dans Rêves enfantins et il n'hésite pas à relater dans Affaire de cœur, le duel qui l'a opposé à Willy, pour l'amour de sa première femme.

19 Henri Focillon, «Éloge de la main», dans Vie des formes, op. cit., p. 110. 
qui n'est pas sans évoquer son passé de caricaturiste; ce que réalise également Robert Lortac au commencement de la Maison automatique. Passé d'illustrateurs, talent de dessinateurs qui deviennent les ressorts du spectaculaire et I'on s'ébahit devant la virtuosité du coup de crayon. De nouveau, la technique est reléguée au second plan car le dispositif est conçu pour faire l'apologie du manuel, soit l'artisanal ou l'artistique, au détriment de l'automatisation 20 . Le trucage image par image, qui manipule le temps, est relégué au second plan, derrière la main qui procède en temps réel. Or, I'animation de dessins, assez rapidement, a été délaissée au profit du papier découpé21. Changement qui n'a pas occasionné pour autant la disparition de la main. Nous la retrouvons en effet, dans deux autres films réalisés par Marius O'Galop, seulement les règles du jeu ont changé. Dans le Lièvre et la Tortue l'animateur joue avec son personnage comme l'enfant s'amuse avec sa poupée, et retrouve ici les gestes de Cohl qui, dans le Songe d'un garçon de café, n'hésitait pas à maltraiter son personnage dessiné. La Tortue nous regarde et le doigt de l'animateur l'oblige à poursuivre I'histoire, comme si elle tentait d'y échapper. Dans Bécassotte à la mer, film en papier découpé, O'Galop de nouveau intervient dans le récit, seulement les mains sont là pour protéger
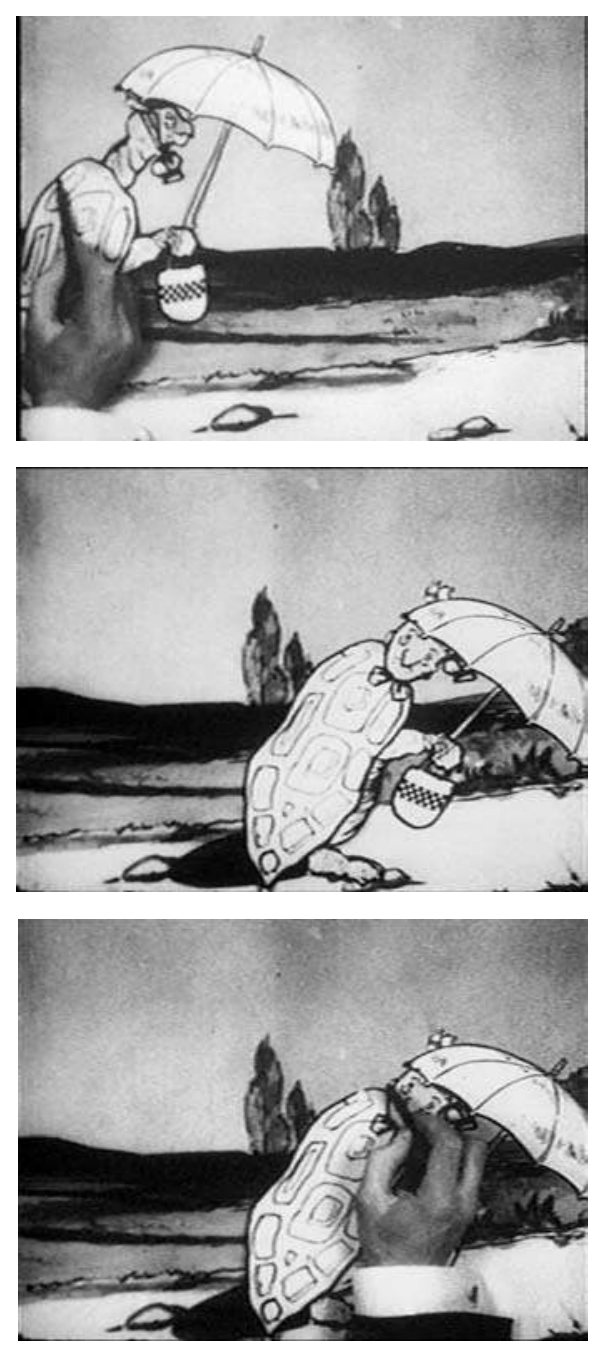

Marius O'Galop, le Lièvre et la Tortue (1921). l'intimité du personnage et le rideau se ferme alors que Bécassotte allait se dévêtir. Rideau de velours, qui clôt la scène en un geste théâtral, alors que dans l'embrasure ainsi pratiquée Bécassotte nous regarde.

20 La critique de la modernité mécanique, telle qu'on la trouve dans la série Mécanicas de Robert Lortac, est peut-être en lien avec ces réflexions.

21 «Peu à peu, pour éviter le long et, il faut bien le dire, fastidieux travail demandé pour la confection de milliers de dessins, on se servit de silhouettes découpées dans du papier bristol et qu'on faisait avancer ou reculer sur le fond après un repérage sévère. Cette intrusion d'un carton rigide amena une véri- 


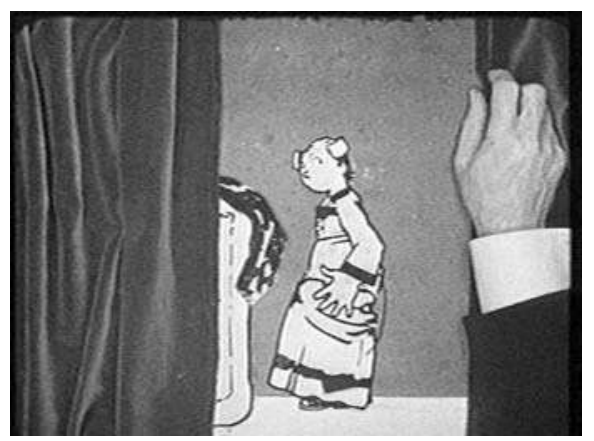

$1895 /$

$n^{\circ} 59$

décembre

2009

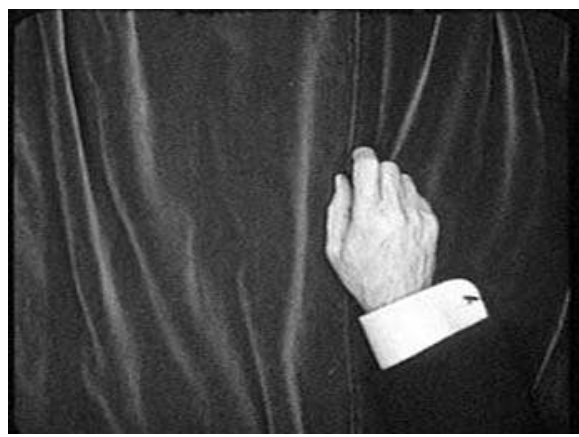

Marius O'Galop, Bécassotte à la mer (1920).
L'animateur s'est donc fait metteur en scène et ces cinéastes par l'entremise des jeux de mains affirment leur désirs d'artistes. Pour Émile Cohl, cette compréhension de l'animation est propre aux cinéastes français:

Hémard, Monnier, Gros, O'Galop, Lortac, Benjamin Rabier ont réalisé des œuvres charmantes dans ce genre de films. Chacun de ces artistes apportant son genre, son esprit, son tour de main qui va de pair avec son tour de manivelle, nous avons chaque fois une œuvre entière qui reflète son auteur; émanation de sa personnalité, de son originale individualité. C'est une consolation pour l'artiste français, à l'amour-propre toujours hypertrophié, et aimant mieux boire dans son verre si petit soit-il qu'accepter de devenir rouage dans une usine à images22.

\section{Éloge du je}

Les cinéastes d'animation français à travers leurs personnages ont créé des univers qui leur appartenaient en propre, et contrairement à ce qu'affirme en 1922, le chroniqueur de Cinémagazine ${ }^{23}$, ils se sont essayés à la création de personnages récurrents: Bécassotte pour O'Galop mais aussi Toto ou Mécanicas pour Lortac. Pourtant ces expériences n'ont pas été continuées; estce pour des raisons industrielles et rencontraient-elles l'adhésion des spectateurs?

Les raisons sont sans doute plus complexes qu'on ne les dira, mais on peut déjà, à partir des témoignages des principaux protagonistes, en évoquer deux. Tout d'abord le cinéma d'animation français ne bénéficie pas des mêmes ressources que le cinéma américain. Et son industrialisation, est sans doute empêchée en raison de la prégnance des paradigmes artistiques

table sécheresse dans le personnage qui, formant une sorte de bloc, bougeait comme un bloc, tout d'une pièce, remuant ses bras, articulés aux épaules, d'un seul tenant, sans les flexions vivantes, si l'on peut dire, qu'on rencontrait dans les dessins faits image par image», J.-B. de Tronquières [Émile Cohl] "Dessins animés ", art. cit.

22 Émile Cohl dans Étienne Arnaud et Boisyvon, op. cit., pp. 84-85. Je souligne.

$23 \mathrm{Cf}$. Lucien Wahl, «La caricature animée. Deux illustres personnages », Cinémagazine, 10 février 1922. 
ou artisanaux déjà évoqués. Émile Cohl ou Robert Lortac, qui eurent l'occasion lors de séjour aux États-Unis d'observer le marché américain, ne manquaient pas de souligner les mérites de la "division du travail» lorsqu'il s'agit d'animer - procédure longue et mécanique qui selon eux ne relève pas de l'art - des dessins ou des objets. En 1922, Cohl décrivait ainsi la situation outre-Atlantique:

Le dessinateur célèbre, créateur du personnage choisi pour passer du journal à l'écran, remet simplement le dessin original qu'il a composé pour l'impression typographique. Puis une équipe de dessinateurs est réunie; ils sont 6, 8, 10 qui se répartissent le travail de «mise en images » en s'inspirant des modèles et des directives de l'artiste chef de bande - c'est le cas de le dire. C'est la division du travail dans toute sa beauté. Dix mains armées de crayons et de pinceaux ont tôt fait d'abattre les croquis nécessaires sans craindre la lassitude. De leur côté les photographes se mettent à photographier les dessins au fur et à mesure qu'ils sortent des mains des croqueurs et ça ronfle! en deux semaines la bande est finie et elle peut être lancée dans le torrent de la circulation cinématographique. Une telle bande revient au moins 20 ou 25000 fr. C'est un prix à faire se trouver mal nos éditeurs français peu enclins par nature à semer aussi libéralement leurs finances. Et voilà pourquoi, ajoute mélancoliquement M. Cohl, les rares et infortunés artistes qui, en France, cultivent ce genre de sport sont à peu près livrés à eux-mêmes. Seuls ils mijotent leurs scénarios, seuls ils dessinent, font leur effarante cuisine artistique, entassent leurs croquis, et les tournent 24 .

Il semble encore, que les personnages développés par les animateurs français relèvent d'un comique "fin de siècle»: Bécassotte est une petite bretonne à qui il arrive des aventures rocambolesques, brimée par une mère peu aimante. Toto ou Touche-à-Tout vit dans un monde à part - ni urbain, ni rural - et ses initiatives, malheureuses, font qu'à chaque épisode, il s'attire les foudres de son père qui ne manque pas de le fesser. Mécanicas (parfois appelé Big Fellow) et son complice Microbus rencontrent des difficultés avec la modernité: voitures agressives, domestiques peu serviables; ils tentent, sans réel succès, de créer un monde à part conforme aux appétits des classes moyennes. Ces personnages sont donc peu glorieux, critiques et asociaux, sans être libertaires ou marginaux (contrairement à Cohl), ce qui, on le devine, ne facilite pas l'identification des spectateurs. De plus, les ressorts comiques reposent volontiers sur l'absurde, en droite ligne de ce qui avait été développé au sein des Incohérents; bohème parfois appelée " Je m'enfoutiste», "Zutistes » ou autres "Hirsutes » 25 et à

24 Émile Cohl dans Étienne Arnaud et Boisyvon, op. cit., pp. 83-84 et 85.

25 Cf. Isabelle Marinone, «Émile Cohl et la bohème » dans Valérie Vignaux (dir.), «Émile Cohl », op. cit., pp. 39-56. 


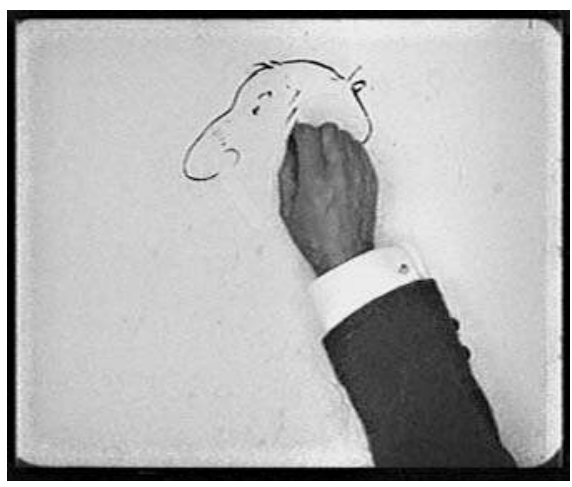

$1895 /$

$n^{\circ} 59$

décembre

2009 laquelle appartenait Émile Cohl. O'Galop s'y réfère explicitement lorsqu'il rend hommage à André Gill dans le Circuit de l'alcool, tandis que Lortac, dans Mécanicas ou la Sève poilifère, nous présente les aventures d'un jeune garçon peu aimable, qui martyrise son entourage, en l'aspergeant d'un produit augmentant la pousse des poils... Or, le cinéma américain est promoteur d'un type social nouveau qui, contrairement au cinéma français, est moins soucieux de moralisme - ou de pédagogie - et où l'impertinence triomphe. Émile Cohl, qui analysait la situation, en 1922, le constatait déjà :

Grâce à leur inépuisables dollars, ils ont monté de véritables entreprises et voici comment ils procèdent: Avant tout, ils choisissent un type populaire bien connu, aimé du public américain. Là-bas, chaque caricaturiste crée un personnage, un fantoche qu'il met à toutes les sauces et qu'il offre chaque samedi dans les formidables suppléments illustrés des journaux. Toutes les semaines c'est une aventure nouvelle plus ou moins cocasse. On ren-

contre là, ce qui nous frappe en France dans les scénarios américains; c'est cette simplicité, cette fraîcheur, cette sorte de candeur d'esprit qui dénote les âmes peu compliquées de la masse du peuple américain. Ainsi le dessinateur Mac Manus a créé le jeune Snokums, un joyeux et désagréable bébé qui en fait voir de cruelles à son pauvre père sous l'œil candide de sa très élégante mère. Ce Snokums a fait, dans son temps son petit stage au cinéma; à son arrivée en France, nous I'appelions Zozor. Un autre artiste, Fisher, qui fut capitaine dans I'armée américaine, pendant la guerre, est le père de Mutt et Jeff devenus ici, on ne sait trop pourquoi Dick et Jeff. Il y a bien d'autres types encore (Happy-Hooligan, les Katzenjammer, etc.) 26.

On le devine, à partir de ces quelques éléments, le cinéma d'animation en France a des singularités qui l'éloignent des succès de masse. Cependant, ces traits constituent son originalité et leurs influences perdurent tout au long de son histoire. On a tenté, en suivant les 
manipulations des animateurs français des premiers temps, d'en découvrir les ressorts, et ainsi on a retrouvé les gestes de l'enfant qui, mis en présence d'un jouet, selon Baudelaire dans «La Morale du Joujou »:

[...] tourne, retourne son joujou, [...] le gratte, le secoue, le cogne contre les murs, le jette par terre. De temps en temps il lui fait recommencer ses mouvements mécaniques, quelquefois en sens inverse. La vie merveilleuse s'arrête. L'enfant, comme le peuple qui assiège les Tuileries, fait un suprême effort; enfin il l'entrouvre, il est le plus fort. Mais où est l'âme ?27.

Les mains des animateurs, parce qu'elles faisaient signes, sont devenues le langage d'une énigme; néanmoins, le mystère demeure entier: Marius $\mathrm{O}^{\prime} \mathrm{Galop}$ on le sait, était ambidextre... 